\title{
Dynamics of polarons in branched conducting polymers
}

\author{
K. K. Sabirov ${ }^{1}$, J. R. Yusupov ${ }^{2}$, Kh. Sh. Matyokubov ${ }^{3}$ \\ ${ }^{1}$ Tashkent University of Information Technologies, 108 Amir Temur Str., 100200, Tashkent Uzbekistan \\ ${ }^{2}$ Turin Polytechnic University in Tashkent, 17 Niyazov Str., 100095, Tashkent, Uzbekistan \\ ${ }^{3}$ Urgench State University, 14 H. Olimjon Str., 220100, Urgench, Uzbekistan \\ karimjonsabirov80@gmail.com
}

PACS 82.35.Cd, 71.38.-k

DOI 10.17586/2220-8054-2020-11-2-183-188

\begin{abstract}
In this paper, we study polarons in branched conducting polymers. For the description of polarons dynamics in such a polymers, we use the modified
\end{abstract} SSH-model in combination with quantum graph concept. Applications and possible extensions of the model are discussed.

Keywords: quantum graph, SSH model, conducting polymers, polaron dynamics.

Received: 20 March 2020

Revised: 3 April 2020

\section{Introduction}

Conducting polymers have attracted much attention in the context of organic electronic and third generation photovoltaics as basic functional materials. Effective practical implementation of such materials requires understanding their electronic properties, especially, charge transport. To solve such a task, one needs to develop highly accurate and realistic models for charge dynamics in conducting polymers. The main charge carriers in conducting polymers are charged solitons, excitons and polarons. The latter is an electron or hole trapped by phonon cloud. Polarons as charge carriers appear in different conducting polymers, when the latter is doped or has defects. The dynamics of polarons in conducting polymers are nicely described within the Su-Schrieffer-Heeger ( $\mathrm{SSH}$ ) model, which is a one dimensional analog of tight-binding models of the solid state physics. Applying the SSH model allows one to calculate band structure and transport characteristics of polarons in conducting polymers. So far, different aspects of charge transport in conducting polymers have been studied within the SSH-model and its modifications (see, Refs. [1-11] for review). However, despite the certain progress made in the study of charge transport in conducting polymers, some aspects of the topic are still far from the research focus area. This mainly concerns branched conducting polymers. These are three or more linear polymer chains connecting to each other at the branching areas. Such polymers have several advantages compared to the linear (unbranched) ones. In particular, by choosing optimal branching architecture, one can tune the functional properties of the materials and devices made of such polymers. Such polymers attracted certain attention over the past decade. A considerable number of papers have been published in the literature during past two decades on the synthesis of different branched polymers and study of their optical, electronic and mechanical properties (see, e.g., [12-29]). A review of the chemistry and physics of hyperbranched polymers is presented in [15]. In [19], a synthesis strategy for a hyperbranched sulfonated polydiphenylamine was developed and electronic properties have been studied. Synthesis and light-emitting applications of several conjugated polymers have been reviewed in [21].

In this paper, we address the problem of polaron dynamics in branched conducting polymers. The system is modeled by means of the quantum graph concept and SSH-model, i.e., by constructing extended version of the latter. This allows one to derive a system of wave equations on metric graphs. We note that the linear wave equations on metric graphs have been extensively studied in the Refs. [30-35] and soliton dynamics in networks has been the topic for extensive research during the past decade (see, e.g., Refs. [36-45]). Modeling such structures in terms of metric graphs provides powerful tool for effective description of the wave dynamics in branched structures appearing in different areas of physics. The graph itself is determined as a set of branches, which are connected to each other at the vertices (branching points) according to some rule. This rule is called the topology of a graph. When branches of a graph are assigned length it is called metric graph. The topology of a graph is given in terms of the adjacency matrix, which is defined, e.g., in [30-34].

The paper is organized as follows. In the next section we briefly recall SSH model on a real line. Section 3 presents extension of the SSH-model on graphs and exact solution of the obtained wave equations. Finally, section 4 presents some concluding remarks. 
a)

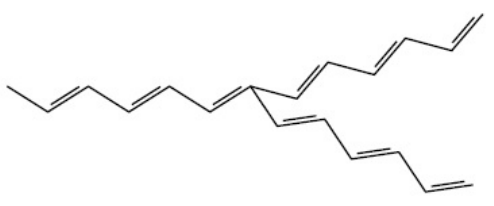

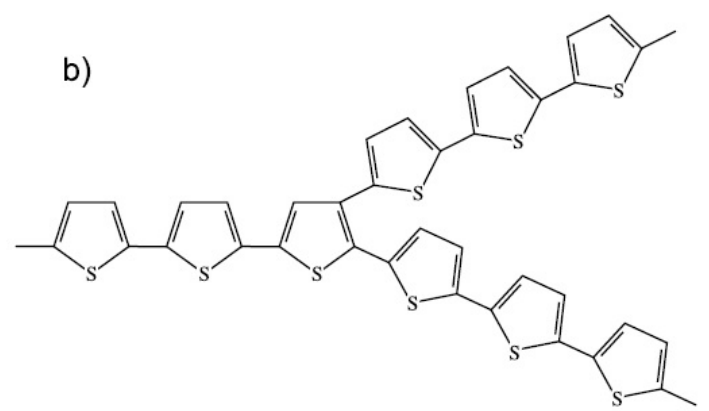

FIG. 1. Sketch of branched conducting polymers

\section{Polaron dynamics on a linear polymers}

Before treating the problem of branched conducting polymers, we briefly recall the linear counterpart of the problem, following the Ref. [6]. The Hamiltonian of SSH model for linear polymer can be written as: [6,7]

$$
\begin{array}{r}
H(t)=\frac{i}{2} \int\left(u(x, t) \partial_{t} u^{*}(x, t)-u^{*}(x, t) \partial_{t} u(x, t)\right) d x+ \\
+\frac{i}{2} \int\left(v(x, t) \partial_{t} v^{*}(x, t)-v^{*}(x, t) \partial_{t} v(x, t)\right) d x+ \\
\quad+\gamma \int\left[\frac{d \Delta(x, t)}{d t}\right]^{2} d x+\theta \int \Delta^{2}(x, t) d x- \\
-i \beta \int\left(u^{*}(x, t) \partial_{x} u(x, t)-v^{*}(x, t) \partial_{x} v(x, t)\right) d x+ \\
+\int \Delta(x, t)\left(u^{*}(x, t) v(x, t)+v^{*}(x, t) u(x, t)\right) d x
\end{array}
$$

where $\beta$ is the effective electron-phonon coupling constant and the pair of the wave functions $u(x, t)$ and $v(x, t)$ describe effective electron-phonon interaction.

The wave equations following from this Lagrangian can be written as:

$$
\begin{gathered}
-i \partial_{t} u+i \beta \partial_{x} u-\Delta(x) v=0, \\
-i \partial_{t} v-i \beta \partial_{x} v-\Delta(x) u=0 .
\end{gathered}
$$

Using substitutions $u(x, t)=\exp (i \epsilon t) u(x), v(x, t)=\exp (i \epsilon t) v(x)$, one can separate time and space variables in these equations:

$$
\begin{array}{r}
\epsilon u=-i \beta \partial_{x} u+\Delta(x) v, \\
\epsilon v=i \beta \partial_{x} v+\Delta(x) u .
\end{array}
$$

Explicit form of the band gap parameter can be written as

$$
\Delta(x)=\Delta_{0}-k_{0} \beta\left[\tanh k_{0}\left(x+x_{0}\right)-\tanh k_{0}\left(x-x_{0}\right)\right] .
$$

For this system of equations the electron wave functions for the positive-energy localized state can be written as $[6,7]$

$$
\begin{gathered}
u_{0}(x)=N_{0}\left[(1-i) \operatorname{sech} k_{0}\left(x+x_{0}\right)+(1+i) \operatorname{sech} k_{0}\left(x-x_{0}\right)\right], \\
v_{0}(x)=N_{0}\left[(1+i) \operatorname{sech} k_{0}\left(x+x_{0}\right)+(1-i) \operatorname{sech} k_{0}\left(x-x_{0}\right)\right],
\end{gathered}
$$

with $N_{0}=\frac{\sqrt{k_{0}}}{4}$. These wave functions are normalized as

$$
\int\left(u_{0}^{2}+v_{0}^{2}\right) d x=1
$$

and $k_{0}$ is found by solving equation $\tanh \left(2 k_{0} x_{0}\right)=k_{0} \beta / \Delta_{0}$. For the above wave functions one can estimate polaron width $x_{0}$ and polaron binding energy $E_{0}[6,7]$ :

$$
2 x_{0} \approx 8.9 a, \quad E_{0}=0.1 \Delta_{0},
$$

where $a$ is the lattice constant. In the next section we extend this model for branched conducting polymer. 


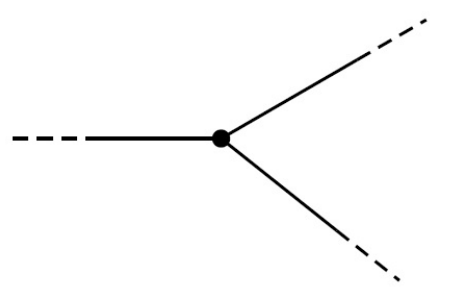

FIG. 2. Star graph

\section{Polarons in branched conducting polymers}

Polarons in conducting polymers appear both as a result of photo-generation, as well as a charge carrier in organic electronic devices when a voltage is applied. An effective way for modeling of polaron dynamics in conducting polymers is the SSH-model, which treats a polymer chain as a 1D lattice and applies nonlinear version of the tightbinding model. Here, we consider dynamics of polarons in a branched conducting polymers consisting of three (linear) polymer chains, which are connected to each other at single monomer (see, Fig.1). The branching is assumed to be in the form of Y-junction (see, Fig.1). Such system can be mapped onto the basic star graph presented in Fig.2. Assuming that the length of polymer is much longer than its width, the dynamics of polaron can be described in terms of the SSH-Hamiltonian given on a star graph with branches $b_{j}, j=1,2,3, b_{1} \sim(-\infty ; 0], b_{2,3} \sim[0 ;+\infty)$. The SSH-Hamiltonian on this star graph is given as:

$$
H=\sum_{j=1}^{3} \int_{0}^{+\infty} H_{j}(t) d t
$$

where:

$$
\begin{aligned}
H_{j}(t)=\frac{1}{\beta_{j}}\left[\frac{i}{2} \int_{b_{j}}\left(u_{j}(x, t) \partial_{t} u_{j}^{*}(x, t)-u_{j}^{*}(x, t) \partial_{t} u_{j}(x, t)\right) d x+\right. \\
+\frac{i}{2} \int_{b_{j}}\left(v_{j}(x, t) \partial_{t} v_{j}^{*}(x, t)-v_{j}^{*}(x, t) \partial_{t} v_{j}(x, t)\right) d x+ \\
\quad+\gamma_{j}\left[\frac{d \Delta_{j}(x, t)}{d t}\right]^{2} d x+\theta_{j} \int_{b_{j}} \Delta_{j}^{2}(x, t) d x- \\
-i \beta_{j}\left(u_{j}^{*}(x, t) \partial_{x} u_{j}(x, t)-v_{j}^{*}(x, t) \partial_{x} v_{j}(x, t)\right) d x+ \\
\left.+\int_{b_{j}} \Delta_{j}(x, t)\left(u_{j}^{*}(x, t) v_{j}(x, t)+v_{j}^{*}(x, t) u_{j}(x, t)\right) d x\right] .
\end{aligned}
$$

Here, $\beta_{j}$ is the effective electron-phonon coupling constant for branch $b_{j}$. Using this Hamiltonian, one can derive wave equations for polaron in branched polymer, as well as boundary conditions at the branching point. The latter is derived from the energy conservation, given by $\dot{H}=0$. Requiring the first variation of (6) should be zero, and using $\delta u_{j}=0, \delta v_{j}=0$ at $t=0$ and $t \rightarrow+\infty, u_{1}, v_{1} \rightarrow 0$ at $x \rightarrow-\infty, u_{2,3}, v_{2,3} \rightarrow 0$ at $x \rightarrow+\infty$ we have wave equations and boundary conditions on the star graph presented in Fig.2:

$$
\begin{array}{r}
-i \partial_{t} u_{j}+i \beta_{j} \partial_{x} u_{j}-\Delta_{j} v_{j}=0, \\
-i \partial_{t} v_{j}-i \beta_{j} \partial_{x} v_{j}-\Delta_{j} u_{j}=0, \\
\left.u_{1}^{*} \delta u_{1}\right|_{x=0}=\left.u_{2}^{*} \delta u_{2}\right|_{x=0}+\left.u_{3}^{*} \delta u_{3}\right|_{x=0}, \\
\left.v_{1}^{*} \delta v_{1}\right|_{x=0}=\left.v_{2}^{*} \delta v_{2}\right|_{x=0}+\left.v_{3}^{*} \delta v_{3}\right|_{x=0} .
\end{array}
$$

Using $u_{j}(x, t)=\exp (i \epsilon t) u_{j}(x), v_{j}(x, t)=\exp (i \epsilon t) v_{j}(x),\left.\frac{1}{\alpha_{1}} \delta u_{1}\right|_{x=0}=\left.\frac{1}{\alpha_{2}} \delta u_{2}\right|_{x=0}+\left.\frac{1}{\alpha_{3}} \delta u_{3}\right|_{x=0}$ and $\left.\alpha_{1} \delta v_{1}\right|_{x=0}=$ $\left.\alpha_{2} \delta v_{2}\right|_{x=0}=\left.\alpha_{3} \delta v_{3}\right|_{x=0}$ one can separate space and time-variables in the wave equation, so that the wave equation 

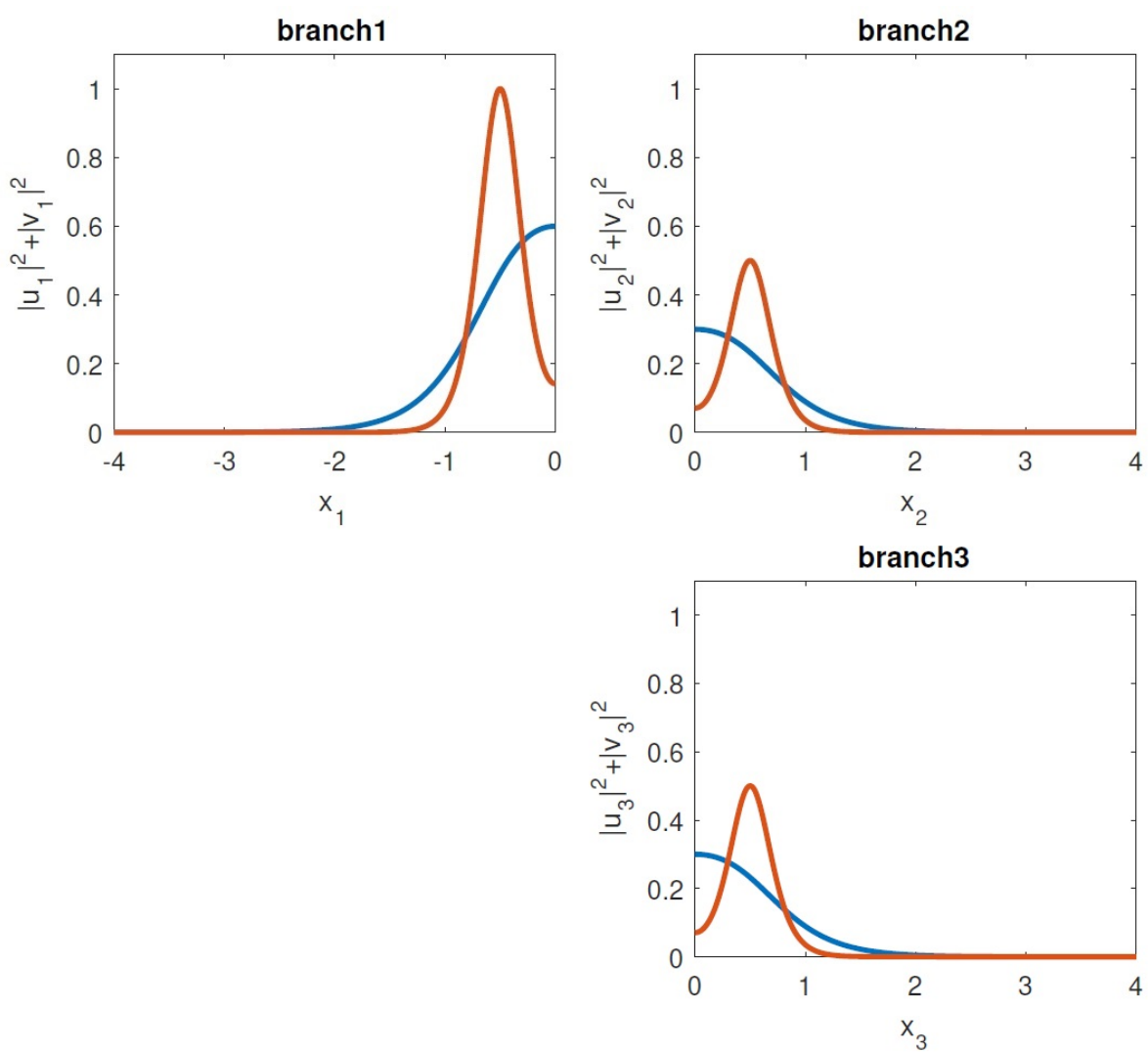

FIG. 3. (Color online) The profile of the squared modulus of the polaron wave function on a branched conducting polymer for the values of $\beta_{j}$ fulfilling the sum rule in Eq.(16): $\beta_{1}=1 ; \beta_{2}=$ $\beta_{3}=\sqrt{2}$. The values of $x_{0}$ and $\sigma$ are chosen as $x_{0}=1 / 3, \sigma=2$ (blue) and $x_{0}=1 / 2, \sigma=4$ (red)

takes the forms:

$$
\begin{array}{r}
\epsilon u_{j}=-i \beta_{j} \partial_{x} u_{j}+\Delta_{j} v_{j} \\
\epsilon v_{j}=i \beta_{j} \partial_{x} v_{j}+\Delta_{j} u_{j}
\end{array}
$$

with:

$$
\begin{aligned}
\left.\alpha_{1} u_{1}\right|_{x=0} & =\left.\alpha_{2} u_{2}\right|_{x=0}=\left.\alpha_{3} u_{3}\right|_{x=0}, \\
\left.\frac{1}{\alpha_{1}} v_{1}\right|_{x=0} & =\left.\frac{1}{\alpha_{2}} v_{2}\right|_{x=0}+\left.\frac{1}{\alpha_{3}} v_{3}\right|_{x=0} .
\end{aligned}
$$

$\alpha_{j}$ are nonzero real constants, which will be determined below. We note that in real applications to polymers they can be chosen on the basis of certain physical properties.

Let us introduce the following notations:

$$
u_{j}(x)=\frac{u_{0}(x)}{\beta_{j}}, v_{j}(x)=\frac{v_{0}(x)}{\beta_{j}} .
$$

Then, exact analytical solution of the problem given by Eqs.(10) and (11) can be written as:

$$
\begin{gathered}
u_{0}(x)=N\left[(1-i) \operatorname{sech} k_{0}\left(x+x_{0}\right)+(1+i) \operatorname{sech} k_{0}\left(x-x_{0}\right)\right], \\
v_{0}(x)=N\left[(1+i) \operatorname{sech} k_{0}\left(x+x_{0}\right)+(1-i) \operatorname{sech} k_{0}\left(x-x_{0}\right)\right], \\
\Delta_{j}(x)=\Delta_{0 j}-k_{0} \beta_{j}\left[\tanh k_{0}\left(x+x_{0}\right)-\tanh k_{0}\left(x-x_{0}\right)\right],
\end{gathered}
$$


where $k_{0}$ is found by solving equation $\tanh \left(2 k_{0} x_{0}\right)=k_{0} / \sigma$, and $\Delta_{0 j}=\sigma \beta_{j}, j=1,2,3$. Requiring that the solutions given by (13) should fulfill the vertex boundary conditions (11), we get the following constraints for $\alpha_{j}$ :

$$
\begin{array}{r}
\frac{\alpha_{1}}{\beta_{1}}=\frac{\alpha_{2}}{\beta_{2}}=\frac{\alpha_{3}}{\beta_{3}}, \\
\frac{1}{\alpha_{1} \beta_{1}}=\frac{1}{\alpha_{2} \beta_{2}}+\frac{1}{\alpha_{3} \beta_{3}},
\end{array}
$$

which make Eq.(13) to be the exact solution of the problem given by: Eqs. (10) and (11):

$$
\frac{1}{\beta_{1}^{2}}=\frac{1}{\beta_{2}^{2}}+\frac{1}{\beta_{3}^{2}},
$$

and $N=\frac{\beta_{1} \sqrt{k_{0}}}{4}$.

The exact solutions given by Eq.(13) present the wave function of polaron in branched conducting polymer having the form of Y-junction. In Fig. 3 the plot of the squared modulus of the wave function is presented for the values of parameters $\beta_{1}=1, \beta_{2}=\beta_{3}=\sqrt{2}$.

\section{Conclusions}

We studied polarons in branched conducting polymers. A model based on the use of a modified SSH-model leading to the linear wave equations system for polarons dynamics is used in combination with quantum graph concept. Explicit wave function of the polaron on branched conducting polymer having Y-junction type branching is obtained. The model proposed in this work can be extended to the case of more complicated branching topologies. Also, it can be applied for modeling of exciton migration in polymer based thin film organic solar cells, where polymer chains packed on the cell create complicated branched structures.

\section{References}

[1] Heeger A.J., and R. Pethig. Charge Storage and Charge Transport in Conducting Polymers: Solitons, Polarons and Bipolarons [and Discussion]. Philosophical Transactions of the Royal Society of London. Series A, Mathematical and Physical Sciences, 1985, 314(1528), P. 17-35.

[2] Heeger A.J. Nobel Lecture: Semiconducting and metallic polymers: The fourth generation of polymeric materials, Rev. Mod.Phys., 2001, 73, P. 681 .

[3] Heeger A.J., Kivelson S., Schrieffer J.R., Su W.-P. Solitons in conducting polymers. Rev. Mod.Phys., 1988, 60, P. 781.

[4] Su W.P., Schrieffer J.R., Heeger A.J. Solitons in Polyacetylene. Phys. Rev. Lett., 1979. 42, P. 1698.

[5] Brazovskii S.A. JETP Lett., 1978, 28, P. 20.

[6] Campbell D.K. and Bishop A.R. Solitons in polyacetylene and relativistic-field-theory models. Phys. Rev. B, 1981, 24, P. 8.

[7] Campbell D.K. and Bishop A.R. Soliton Excitations in Polyacetylene and Relativistic Field Theory Models. Nuclear Physics B, 1982, 200[FS4], P. 297-328.

[8] Horovitz B. Soliton Lattice in Polyacetylene. Spin-Peierls Systems, and Two-Dimensional Sine-Gordon Systems. Phys. Rev. Lett., 1981, 46, P. 11.

[9] Horovitz B. The mixed peierls phase and metallic polyacetylene. Solid State Communications, 1980, 34, P. 61-64.

[10] Horovitz B. Solitons in polyacetylene: A comment. Phys. Rev. B., 1980, 22, P. 2.

[11] Krumhansl J.A., Horovitz B., and Heeger A.J. Amplitude solitons in incommensurate Peierls systems: Implications for TTF-TCNQ. Solid State Communications, 1980, 34, P. 945-948.

[12] Wallace D.S., Stoneham A.M., Hayes W., Fisher A.J. and Testa A. Theory of defects in conducting polymers. II. Application to polyacetylene. J. Phys.: Condens. Matter, 1991, 3, P. 3905.

[13] Fujii M., Ari K. and Yoshino K. Branching Patterns of a Conducting Polymer Polymerized Electrochemically with a ConstantCurrent Source, J. Electrochem. Soc., 1993, 140, P. 7.

[14] Jurkiewicz J. and Krzywicki A. Branched polymers with loops. Phys. Lett. B, 1997, 392, P. 291-297.

[15] Inoue K. Functional dendrimers, hyperbranched and star polymers. Prog. Polym. Sci., 2000, 25, P. 453.

[16] Dai L., Winkler B., Dong L., Tong L. and Mau A.W.H. Conjugated Polymers for LightEmitting Applications, Adv. Mater., 2001 , 13, P. 12-13.

[17] Wu D.T., Synth. Met.. Theory for conductivity in conducting star polymer blends, 2002, 126, P. 289.

[18] Gao C. and Yan D. Hyperbranched polymers: from synthesis to applications. Prog. Polym. Sci., 2004, 29, P. 183.

[19] Hua F. and Ruckenstein E. Hyperbranched Sulfonated Polydiphenylamine as a Novel Self-Doped Conducting Polymer and Its pH Response. Macromolecules, 2005, 38, P. 888.

[20] Ch. Wu, Malinin S.V., Tretiak S., Chernyak V.Y. Exciton scattering and localization in branched dendrimeric structures. Nature Physics, 2006, 2, P. 631-635.

[21] Lee R.-H., Chen W.-Sh., and Wang Y.-Y. Synthesis and electroluminescence properties of a series of hyper-branched light-emitting polymers. Thin Solid Films, 2009, 517, P. 5747.

[22] Hirao A. and Yoo H.-S. Dendrimer-like star-branched polymers: novel structurally well-defined hyperbranched polymers. Polymer J., 2011, 43, P. 2.

[23] Hutchings L.R. Complex Branched Polymers for StructureProperty Correlation Studies: The Case for Temperature Gradient Interaction Chromatography Analysis. Macromolecules, 2012, 45, P. 5621.

[24] Pan L., Yu G., Zhai D., et.al. Hierarchical nanostructured conducting polymer hydrogel with high electrochemical activity. Proc. Natl. Acad. Sci. U.S.A., 2012, 109, P. 9287. 
[25] Li H., Malinin S.V., Tretiak S., and Chernyak V.Y. Effective tight-binding models for excitons in branched conjugated molecules. J. Chem. Phys., 2013, 139, P. 064109.

[26] Ohshita J., Tominaga Y., Tanaka D., Mizumo T., Fujita Y., Kunugi Y. Synthesis and optical properties of organosiliconoligothiophene branched polymers. J. Org. Chem., 2013, 50, P. 736.

[27] Otrokhov G.V., Morozova O.V., Vasileva I.S., Shumakovich G.P., Zaitseva E.A., Khlupova M.E., and Yaropolov A.I. Biocatalytic synthesis of conducting polymers and prospects for its application. Biochemistry, 2013, 78, P. 1539.

[28] Goll M., Ruff A., Muks E., Goerigk F., Omiecienski B., Ruff I., Gonzalez-Cano R. C., Navarrete J. T. L., Delgado M. C. R. and Ludwigs S. Functionalized branched EDOT-terthiophene copolymer films by electropolymerization and post-polymerization click-reactions. Beilstein J. Org. Chem., 2015, 11, P. 335.

[29] Soganci T., Gumusay O., Soyleyici H. C., Ak M. Synthesis of highly branched conducting polymer architecture for electrochromic applications, Polymer, 2018, 134, P. 187.

[30] Kostrykin R., Schrader R., Kirchhoffs rule for quantum wires. J. Phys. A: Math. Gen., 1999, 32, P. 595.

[31] Kottos T. and Smilansky U. Periodic Orbit Theory and Spectral Statistics for Quantum Graphs. Ann.Phys., 1999, 274(1), P. 76-124.

[32] Kuchment P. Waves in Random Media, 2004, 14 P. S107.

[33] Gnutzmann S. and Smilansky U. Quantum graphs: Applications to quantum chaos and universal spectral statistics. Adv.Phys., 2006, 55(5-6), P. 527-625.

[34] Exner P. and Kovarik H. Quantum waveguides. Springer, 2015.

[35] Gnutzmann S., Keating J.P. and Piotet F. Eigenfunction statistics on quantum graphs. Ann.Phys., 2010, 325(12) P. 2595-2640.

[36] Sobirov Z., Matrasulov D., Sabirov K., Sawada S., and Nakamura K. Integrable nonlinear Schrdinger equation on simple networks: Connection formula at vertices. Phys. Rev. E, 2010, 81, P. 066602.

[37] Sobirov Z., Matrasulov D., Sawada S., and Nakamura K. Transport in simple networks described by an integrable discrete nonlinear Schrdinger equation. Phys.Rev.E, 2011, 84, P. 026609.

[38] Adami R., Cacciapuoti C., Finco D., Noja D. Fast Solitons on Star Graphs. Rev.Math.Phys, 2011, 23 , P. 4.

[39] Sabirov K.K., Sobirov Z.A., Babajanov D., and Matrasulov D.U. Stationary nonlinear Schrödinger equation on simplest graphs. Phys.Lett. A, 2013, 377, P. 860.

[40] Caputo J.-G., Dutykh D. Nonlinear waves in networks: Model reduction for the sine-Gordon equation. Phys. Rev. E, 2014,90 , P. 022912.

[41] Uecker H., Grieser D., Sobirov Z., Babajanov D. and Matrasulov D. Soliton transport in tubular networks: Transmission at vertices in the shrinking limit. Phys. Rev. E, 2015, 91, P. 023209.

[42] Noja D. Nonlinear Schrödinger equation on graphs: recent results and open problems. Philos. Trans. R. Soc. A, 2014, 372, P. 20130002.

[43] Noja D., Pelinovsky D., and Shaikhova G. Bifurcations and stability of standing waves in the nonlinear Schrödinger equation on the tadpole graph. Nonlinearity, 2015, 28, P. 2343.

[44] Sobirov Z., Babajanov D., Matrasulov D., Nakamura K., and Uecker H. Sine-Gordon solitons in networks: Scattering and transmission at vertices, EPL, 2016, 115, P. 50002.

[45] Sabirov K.K., Rakhmanov S., Matrasulov D. and Susanto H. The stationary sine-Gordon equation on metric graphs: Exact analytical solutions for simple topologies. Phys.Lett. A, 2018, 382, P. 1092. 